\title{
Acessibilidade em ambiente rural: uma abordagem multicritério com uso de SIG
}

\author{
Elisa Sakamoto ${ }^{1}$ e Josiane Palma Lima²
}

\begin{abstract}
Resumo: O presente trabalho tem por objetivo apresentar a avaliação da acessibilidade no meio rural, de produtores familiares, por meio de um estudo de caso no Município de Itajubá, MG. A agregação do Método Hierárquico Analítico (AHP) a um Sistema de Informação Geográfica permitiu a geração de mapas de acessibilidade, tanto para cada grupo de Locais de Interesse - LI (Comercialização da produção, Ensino, Saúde, Serviços e Lazer), quanto de forma global para todo território. O estudo considerou o grau de importância dos locais, as distâncias percorridas, tipo de pavimento das vias e acesso ao transporte público, levando em consideração as características da região, a opinião dos produtores e especialistas. Os locais de comercialização da produção foram considerados pelos especialistas como os mais importantes na avaliação da acessibilidade, e os mapas gerados possibilitaram verificar as áreas com maior e menor acessibilidade, e assim, as áreas prioritárias para planejamentos e investimentos em infraestrutura e transportes.

Palavras-chave: Acessibilidade; Análise Multicritério; Desenvolvimento Rural; Sistemas de Informações Geográficas.
\end{abstract}

Abstract: This study to present accessibility assessment in rural areas and a case study at Itajubá city, state of Minas Gerais, Brazil. The aggregation of Hierarchical Analytic Process (AHP) to a Geographic Information System allowed the generation of accessibility maps, for each group of Places of Interest (Trade of Production, Education, Health, Services and Leisure) and for whole territory. The study considered the level of importance of the places, the distances, the type of pavement used on the roads and the access to the public transportation, considering the characteristics of the region, the opinion of the growers and specialists. The trade of production was considered by the specialists the most important on the evaluation of accessibility and the maps show the areas with more and less accessibility, the priority areas for planning and investments on infrastructure and transportation.

Keywords: Accessibility; Multicriteria Analysis; Rural Development; Geographic Information Systems.

\section{INTRODUÇÃO}

Os espaços urbanos normalmente contam com processos de planejamento com uso frequente de métodos de tomada de decisões, envolvendo vários fatores, critérios e objetivos. E quando se pensa o desenvolvimento sustentável das cidades, o estudo da acessibilidade e os fatores relacionados se tornam fundamentais, pois propiciam o deslocamento eficiente das pessoas e cargas no espaço urbano (Tobias et al., 2012).

Nesse sentido, considera-se acessibilidade essencialmente como a facilidade de se atingir os destinos desejados (Vasconcellos, 2000). Sendo posta pela relação entre o transporte, o uso do solo e habilidades individuais, como uma medida de esforço para que uma separação espacial possa ser transposta. E assim, possibilitar o acesso das pessoas aos espaços e equipamentos para exercer suas diversas atividades, tais como educação, saúde, trabalho, lazer etc. (Raia, 2000). Portanto, a acessibilidade deve ser vista como parte integrante de uma política de mobilidade urbana, pois está relacionada à possibilidade do exercício de direitos das pessoas, a inclusão social e a uma maior equiparação de oportunidades (Ministério das Cidades, 2006).

\footnotetext{
Elisa Sakamoto, Universidade Federal de Itajubá (sakamoto.elisa@gmail.com).

2 Josiane Palma Lima, Universidade Federal de Itajubá, Instituto de Engenharia de Produção e Gestão (jplima@unifei.edu.br).
}

Manuscrito recebido em 29/06/2015 e aprovado para publicação em 11/02/2016.

Este artigo é parte de TRANSPORTES v. 24, n. 1, 2016 ISSN: 2237-1346 (online). DOI: 10.14295/transportes.v24i1.958
De acordo com Rodrigues (2001), os estudos sobre a acessibilidade são discutidos no contexto urbano há aproximadamente dois séculos, entretanto, em relação aos espaços rurais esses estudos não acontecem na mesma medida. $\mathrm{O}$ espaço rural, apesar de apresentar baixa densidade demográfica e população dispersa, apresenta dificuldades específicas de acesso, tanto em relação à produção agropecuária, inclusive da agricultura familiar, como o acesso de toda comunidade rural a bens e serviços básicos, como educação, saúde, transporte público, etc. Dessa forma, a acessibilidade aos diversos locais de interesse e necessidades contribui para reduzir as diferenças de oportunidades existentes nos espaços urbano e rural.

Neste contexto, ao realizar um estudo de acessibilidade no meio rural, o presente trabalho aborda a localização de propriedades rurais com produção familiar, no que se refere ao conceito de multifuncionalidade, que entende a Agricultura Familiar como fonte de trabalho e renda no meio rural, tendo em vista não apenas o componente econômico da produção agropecuária, mas também abordando aspectos sociais, ambientais, culturais e de segurança alimentar, fundamentais nos processos de desenvolvimento do território (Maluf, 2002).

Segundo IFAD (2010), apesar do significativo progresso mundial na redução da pobreza nas duas últimas décadas, o desempenho da agricultura familiar é fundamental na erradicação da fome no campo, já que no mundo $70 \%$ das pessoas que vivem em nível de subsistência se encontram em áreas rurais e, a falta de acessibilidade pelas estradas rurais tem sido identificada como uma das principais causas da pobreza entre a população rural (Lebo e Schelling, 2001). Dessa forma, a população rural fica segregada 
social e espacialmente, pois no meio rural, geralmente, não possuem infraestrutura e meios de transportes adequados para realizar os deslocamentos, principalmente devido a não proximidade aos equipamentos e serviços públicos (Pegoretti, 2006).

Portanto, o objetivo deste trabalho é apresentar a avaliação da acessibilidade de produtores familiares, por meio de um estudo de caso no Município de Itajubá, MG. O método de cálculo do índice de acessibilidade integra a metodologia de análise multicritério em um Sistema de Informação Geográfica (SIG). A pesquisa aborda a agricultura familiar por sua relevância e importância local, pois segundo o IBGE (2009), Itajubá possui 866 estabelecimentos familiares rurais, correspondendo a $87,8 \%$ dos estabelecimentos agropecuários no município, que abastecem parte da demanda local de alimentos, sendo também uma importante fonte de renda e trabalho local.

\section{DESENVOLVIMENTO RURAL E AGRICULTURA FAMILIAR}

As questões envolvendo o Desenvolvimento Rural são estudadas a partir da noção de territórios, e tornou-se base para concepção de diversas políticas públicas que abrangem aspectos econômicos, sociais, culturais e ambientais, de forma a promover um desenvolvimento mais sustentável no território (MDA/SDT, 2003). Dessa forma, é possível verificar muitos trabalhos que abordam a dicotomia rural e urbana em conjunto com o conceito de territórios (Abramovay, 1998; Kageyama, 2004; Veiga, 2007; Miranda, 2011), em que compreendem tanto o ambiente rural quanto o urbano de uma dada localidade, explicitando que os dois ambientes não estão desvinculados nos processos de planejamento de desenvolvimento e não podem ser compreendidos separadamente, pois as relações entre ambos se dão de forma cada vez mais integrada.

Ao associar o desenvolvimento à dimensão territorial, passa-se a reconhecer as capacidades locais como fatores de influência do desenvolvimento, principalmente no que diz respeito ao protagonismo social dos atores locais, como afirmam as diversas teorias sobre capital social, governança local, desenvolvimento local e endógeno, trazendo à tona a importância da agricultura familiar (Sachs, 2002; Abramovay, 2003). Os parâmetros com relação à agricultura familiar se baseiam na Lei $\mathrm{n}^{\circ} 11.326$ (BRASIL, 2006), que permitiu uma delimitação conceitual necessária inclusive para obtenção de informações oficiais e para as políticas públicas relacionadas ao desenvolvimento rural.

De forma geral, a agricultura familiar é uma forma de produção agrícola normalmente diversificada, em que predomina a gestão e o trabalho familiar em pequenas áreas, sendo a renda da família proveniente principalmente desse trabalho. Nesse sentido, o desenvolvimento rural depende de vários fatores que, de certa forma, se relacionam com o meio urbano. $\mathrm{O}$ acesso das comunidades rurais a bens e serviços localizados tanto no meio rural quanto no urbano, é um dos principais fatores para garantir o direito à cidadania e assegurar boas condições de vida nesses espaços. Segundo Santos (2008), é possível estabelecer uma relação entre falta de acessibilidade e a incidência de pobreza e exclusão social, tanto em grandes centros urbanos, quanto em zonas rurais, pois em ambos os espaços há a presença de formas variadas de desvantagens de localização, assim a melhoria da acessibilidade pode contribuir para o aumento das oportunidades de forma mais equitativa no território.

\section{AVALIAÇÃO MULTICRITÉRIO DA ACESSIBILIDADE}

O conceito de acessibilidade e seus aspectos vêm sendo discutido há muitos anos por vários autores. Segundo Oliveira e Silva (2015), tanto a acessibilidade quanto a mobilidade e a capacidade ambiental são vistos como aspectos centrais de sustentabilidade para o novo paradigma de planejamento de transporte. Sob essa nova perspectiva, há uma associação entre o transporte público, a circulação e o planejamento das atividades urbanas, tendo em vista o planejamento para uma mobilidade sustentável.

Inicialmente, Ingram (1971) definiu acessibilidade como sendo a característica (ou vantagem) inerente a um local no que diz respeito a vencer alguma forma de resistência ao movimento. Morris et al., (1979) apresenta uma classificação e formulação de medidas de acessibilidade relativa e acessibilidade integral. Na última, inclui medidas de separação entre todos os pontos de uma rede, medidas de separação incorporando a influência da distância, restrições de capacidade na rede e medidas compostas de separação e oferta/procura. A classificação proposta por Morris et al., (1979) abriu caminho para vários outros autores definirem medidas de acessibilidade de acordo com objetivos específicos a alcançar e de formas muito diversificadas (ver Allen et al., 1993; Mendes, 2001; Aguiar, 2010; Machado e Lima, 2015).

O estudo desenvolvido concentra-se na avaliação dos níveis de acessibilidade para uma determinada área territorial, considerando os Locais de Interesse (LI) para onde os produtores rurais e seus familiares normalmente se deslocam, e as dificuldades à mobilidade encontradas ao longo do percurso. Os locais de interesse foram hierarquizados e agrupados de acordo com as necessidades dos produtores e, determinadas pelo motivo da viagem e a funcionalidade do local. Assim, para a avaliação da acessibilidade admite-se que:

- A acessibilidade avalia-se por objetivo, neste caso, a necessidade de se deslocar da propriedade rural até um determinado LI;

- O índice de acessibilidade é uma medida que incorpora o efeito de três atributos: distância do percurso entre as propriedades rurais e os LI, características da rede (pavimentada ou não) e distância das propriedades ao transporte público;

- Os LI são caracterizados pelo objetivo/propósito em se deslocar, possuindo importâncias diferenciadas (pesos dos LI);

- Os LI são alcançados através da rede viária existente, podendo os seus segmentos apresentar níveis de impedância diferenciados (por exemplo, as características das vias);

- As distâncias-custo resultam da aplicação da impedância às distâncias medidas ao longo da rede da propriedade ao LI, somada às distâncias da propriedade ao transporte público; 
- O índice de acessibilidade de cada propriedade rural resulta da soma ponderada das distâncias-custo normalizadas, através de funções fuzzy, aos LI considerados.

A forma adotada para a quantificação da importância dos vários LI, ou seja, a sua importância na avaliação da acessibilidade e a forma de agregação, tem por base a metodologia multicritério (MCDA) de avaliação da acessibilidade por meio do Processo Hierárquico Analítico - AHP (Saaty, 2008) proposta no trabalho de Mendes (2001), sendo adaptada ao objetivo desta pesquisa. No modelo admite-se que os LI funcionam como critérios na avaliação da acessibilidade, ou seja, possuem importâncias distintas que serão traduzidas em pesos diferenciados no valor final do índice de acessibilidade.

Contribuíram para o cálculo do índice, os valores normalizados de distância-custo. Segundo Lima (2009), para que os valores dos diferentes critérios possam ser agregados é necessário efetuar a sua normalização. O processo de normalização é essencialmente idêntico ao processo de fuzzification colocado pela lógica em conjuntos fuzzy, em que critérios contínuos são normalizados recorrendo-se a funções fuzzy, segundo as quais um conjunto de valores expresso numa dada escala é convertido num outro, comparável e expresso numa escala normalizada (neste trabalho, de 0 a 1). Um dos aspectos críticos desta etapa é a seleção dos pontos de controle utilizados para calibrar a função fuzzy, considerando critérios e realidades particulares. Também é possível incorporar diversas formas de funções fuzzy ao processo, utilizadas para orientar a variação entre o ponto mínimo e o valor máximo, sendo as mais conhecidas: sigmoidal, j-shaped, linear e complexa (Para mais informações sobre procedimentos de normalização por meio de funções fuzzy ver Rodrigues et al., 2004 e Lima, 2009).

Por sua vez, o índice de acessibilidade $A_{i}$ é resultado de uma avaliação multicritério da localização de origens $i$ aos grupos de locais de interesse $j$, dado por uma normalização fuzzy das distâncias-custo $f\left(\mathrm{c}_{i j}\right)$, aplicando os pesos dos LI $\left(w_{j}\right)$. Os pontos $i$, para os quais a acessibilidade é avaliada, dependem da forma como o espaço é modelado e para este trabalho se tratam das propriedades rurais.

$$
A_{i}=\Sigma_{j} f\left(c_{i j}\right) w_{j}
$$

Em que:

$A_{i}=$ índice de acessibilidade em cada propriedade rural $(i)$;

$c_{i j}=$ valores de distância-custo

$f\left(c_{i j}\right)=$ Normalização das distâncias-custo por funções fuzzy $w_{j}=$ Pesos dos locais de interesse $(j)$

Segundo Rodrigues (2001), é possível avaliar a acessibilidade de cada local analisado em relação a um grupo (g) de locais de interesse, assim, os vários destinos (LI) em análise podem ser complementares ou equivalentes, podese considerar então, o seu agrupamento de acordo com suas funcionalidades e dessa forma, o índice passa a ser avaliado por grupos, conforme a equação (2) e (3).
$A_{l}^{g}=\sum_{j}^{n} f\left(c_{i j}\right) w_{j}^{g}$

$A_{i}=\sum_{g} A_{l}^{g} w_{g}$

\section{Em que:}

$A_{l}^{g}$ - Índice de acessibilidade do local $i$ em relação ao grupo $g$

$f\left(c_{i j}\right)$ - Função fuzzy aplicada à distância-custo entre o local $i$ e o destino-chave $j$ incluído no grupo $g$

$w_{j}^{g}$ - Peso do destino-chave $j$ incluído no grupo $g$

$n_{g}$ - Número de destino-chave do grupo $g$

As equações 1, 2 e 3 correspondem à combinação linear ponderada (WLC, Weighted Linear Combination) que permitem calcular o índice de acessibilidade por grupo de destinos e global, compensando as qualidades entre os critérios (trade off). Portanto, o índice de acessibilidade global proposto é uma combinação ponderada linear dos grupos de locais de interesse.

\section{METODOLOGIA}

A metodologia utilizada neste trabalho parte de um estudo quantitativo sobre a acessibilidade em território rural e aborda um estudo de caso realizado com agricultores familiares no município de Itajubá-MG.

As etapas utilizadas seguem a proposta do Processo Hierárquico Analítico (AHP) com as fases cruciais num processo de tomada de decisão, por meio da estruturação do problema com a definição dos locais de interesse, passando pela ponderação e normalização dos valores e, por fim, a combinação de todos os fatores considerados na análise, conforme é apresentado na Figura 1 e depois tratado na próxima seção.

\section{ESTUDO DE CASO: ACESSIBILIDADE NA ZONA RURAL DE ITAJUBÁ - MG}

O município de Itajubá está localizado no sul do estado de Minas Gerais, na Serra da Mantiqueira, a $261 \mathrm{~km}$ de São Paulo, 318 km do Rio de Janeiro e 445 km de Belo Horizonte. Possui 90.658 habitantes, sendo que apenas 8.71\% são residentes rurais, o que configura uma população tipicamente urbana, contudo, a área rural representa mais de 70\% do total da área municipal (IBGE, 2010) em que é significativa a presença da agricultura familiar.

O método de avaliação multicritério da acessibilidade foi escolhido por abordar aspectos de localização, impedância ao deslocamento e a importância que pode ser atribuída a cada um dos locais de interesse, o que possibilita uma análise mais realista do território. Assim, o trabalho pode ser dividido em duas etapas:

- Estudo do perfil dos produtores rurais com a aplicação de uma entrevista em que se verificou a produção agropecuária e também os hábitos e padrões de viagens 


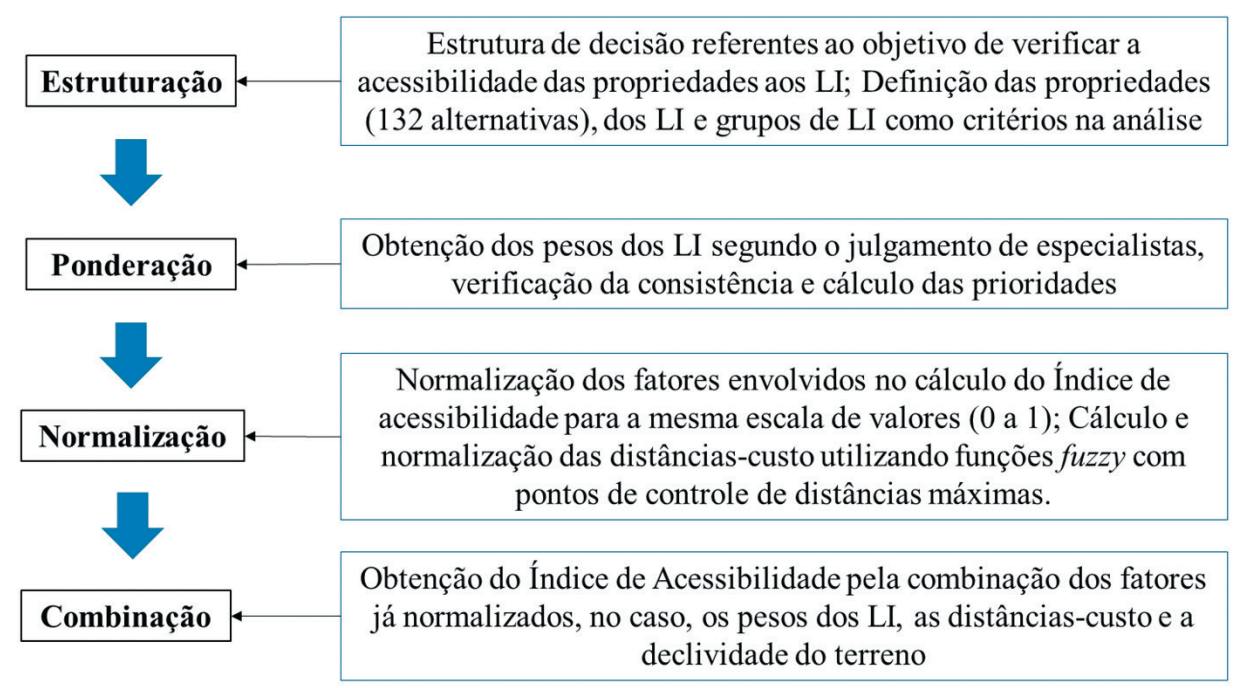

Figura 1: Etapas para Avaliação da Acessibilidade em Território Rural

de toda a família, o que permitiu estabelecer uma lista dos principais locais de interesse dos agricultores familiares em termos de comercialização da produção, educação, saúde, serviços e lazer;

- Avaliação da acessibilidade na região rural do município, que contou primeiramente com a delimitação dos LI e seus agrupamentos de acordo com a sua funcionalidade, passando para a definição do grau de importância dos LI, o cálculo das distâncias-custo e o cálculo do índice de acessibilidade. Depois foram gerados os mapas de acessibilidade por grupos de LI e de forma global para todos os LI em ambiente SIG, considerando posteriormente a declividade como fator de fricção da superfície do terreno.

\subsection{Estrutura de decisão: definição dos locais de interesse}

A coleta de dados em campo contou com o uso de um aparelho GPS portátil, que permitiu mapear um total de 132 propriedades da agricultura familiar, em que foram realizadas entrevistas semiestruturadas com a aplicação de questionário com os produtores familiares, que teve como objetivo obter informações de caracterização (propriedade familiar/área/ mão de obra/ fonte de renda/tipo de produção etc.) e sobre comportamentos, opiniões e a percepção do produtor para questões relacionadas com problemas enfrentados no seu dia a dia e a acessibilidade das propriedades familiares.

A partir dos dados obtidos, foi possível verificar que na região predominam as pequenas propriedades e minifúndios em que a mão de obra é essencialmente familiar, e apesar de algumas famílias realizarem outras atividades ou trabalhos, a produção agropecuária é a principal fonte de renda. A pecuária leiteira e a horticultura são as produções mais expressivas, sendo mais comum a comercialização e abastecimento da demanda local, dentro do próprio município. Apesar da existência de intermediários, a venda direta em laticínios, CEASA (Central Estadual de Abastecimento S/A), feiras e supermercados são as mais utilizadas.

Os LI foram definidos a partir de uma lista preliminar dos locais mais frequentados pelos produtores rurais e seus familiares, sendo estes agrupados com base nas ca- racterísticas e comportamento dos produtores e sua família conforme o motivo da viagem, ou seja, sua funcionalidade: para a comercialização da produção, para educação, para saúde, lazer, bens ou serviços. Dessa forma, os LI dos produtores entrevistados funcionam no modelo de avaliação como critérios de análise.

\subsection{Ponderação: definição dos graus de importância de acordo com a sua funcionalidade}

A avaliação do grau de importância dos LI foi realizada pelo método AHP, por meio de matrizes de comparação par a par proposta por Saaty (2008). Utilizou-se a escala de nove dígitos, possibilitando incorporar na análise diferentes pontos de vistas e a análise de consistência dos dados (para mais detalhes ver Malczewski, 1999; Lima, 2007). O trabalho contou com dez especialistas em agricultura familiar e desenvolvimento rural, ou seja, pessoas que possuem uma relação próxima com a realidade da pesquisa: professores e pós-graduandos da Universidade Federal de Itajubá, especialistas ou profissionais da Empresa de Assistência Técnica e Extensão Rural, Secretaria de Agricultura, Secretaria de Assistência Social, Instituto Brasileiro de Geografia e Estatística e membros do Conselho Municipal de Desenvolvimento Sustentável Rural.

Depois da avaliação realizada pelos especialistas, foi possível calcular os pesos de cada LI que serviram para a avaliação da acessibilidade das propriedades e da área rural. Os especialistas foram ainda questionados quanto às distâncias máximas (Dmáx) que consideram adequadas para o deslocamento dos produtores rurais e seus familiares aos vários LI e ao transporte público. Os valores de Dmáx foram obtidos a partir da média aritmética das 10 avaliações, e foram utilizados na normalização dos valores de distâncias aos LI (DLI). Os locais de interesse com os respectivos agrupamentos, os pesos calculados e as distâncias máximas aos LIs são apresentados na Tabela 1 .

Observou-se que tanto na obtenção dos julgamentos dos especialistas, quanto ao grau de importância dos LI como nos valores de Dmáx, os especialistas buscavam um parâmetro de comparação para as respostas, com isso suas experiências foram incorporadas em suas análises. Assim, as avaliações tenderam à realidade dos entrevistados e suas vivências. 
Tabela 1: Pesos dos LI e grupo de LI e distâncias máximas aos LI

\begin{tabular}{|c|c|c|c|c|c|}
\hline Grupo de LI & $\begin{array}{l}\text { Peso do } \\
\text { grupo }\end{array}$ & $L I$ & $\begin{array}{l}\text { Peso dos } \\
\text { locais }\end{array}$ & $\begin{array}{l}\text { Dmáx } \\
(\mathrm{km})\end{array}$ & $\begin{array}{l}\text { Quantidade de } \\
\text { Pontos por LI }\end{array}$ \\
\hline \multirow{5}{*}{$\begin{array}{l}\text { 1. Comercialização da Produção } \\
\text { agropecuária }\end{array}$} & \multirow{5}{*}{0.376} & Laticínios & 0.309 & 30 & 8 \\
\hline & & Frigorífico & 0.070 & 35 & 1 \\
\hline & & CEASA & 0.239 & 25 & 1 \\
\hline & & Feiras & 0.240 & 25 & 2 \\
\hline & & Supermercados & 0.143 & 25 & 4 \\
\hline \multirow{2}{*}{ 2. Equipamentos de Saúde } & \multirow{2}{*}{0.365} & Hospitais & 0.579 & 20 & 3 \\
\hline & & Postos de saúde (UBS) & 0.421 & 10 & 7 \\
\hline \multirow{3}{*}{ 3. Instituições de Ensino } & \multirow{3}{*}{0.198} & Escolas Rurais (E.F) & 0.393 & 5 & 7 \\
\hline & & $\begin{array}{l}\text { Escolas Urbanas (E. } \\
\text { F/E.M.) }\end{array}$ & 0.403 & 15 & 18 \\
\hline & & IES (E.T/E.S) & 0.204 & 15 & 8 \\
\hline \multirow{2}{*}{ 4. Locais de Serviços e Lazer } & \multirow{2}{*}{0.061} & Serviços e Comércio & 0.458 & 20 & 1 \\
\hline & & Supermercados & 0.542 & 20 & 4 \\
\hline
\end{tabular}

Dmáx ao transporte público $2,5 \mathrm{~km}$

O grupo de LI que recebeu maior importância foi o de "Comercialização da Produção", que se refere aos estabelecimentos de comercialização da produção agropecuária, o que pode ser justificado pelo fato da renda depender em grande parte da produção agropecuária para o sustento familiar, com maior regularidade e frequência dos deslocamentos, pois os produtos comercializados geralmente são perecíveis. Os locais destinos de comercialização como laticínios, feiras, CEASA e supermercados receberam maiores importâncias pelos especialistas, tanto por serem mais representativos na região devido à grande parte da produção ser comercializada nestes locais (hortícolas e leite) quanto pela maior frequência de comercialização, nestes casos mais de uma vez na semana, enquanto que nos demais locais a comercialização costuma ser mais esporádica.

Em segundo lugar em grau de importância foi considerado o grupo "Equipamentos de Saúde", que apesar dos deslocamentos a este tipo de local ocorrer de forma mais pontual ou quando necessário, os especialistas entendem que este tipo de serviço público é essencial e básico para a vida da população no meio rural. Os postos de saúde rurais foram considerados menos importantes que hospitais, porque apesar de serem os locais em que primeiro a comunidade rural costuma receber atendimentos, consultas diversas e encaminhamentos, somente nos hospitais ocorrem os atendimentos com infraestrutura adequada e a maioria dos exames que se fazem necessários.

Com relevância na análise, mas em terceiro lugar em grau de importância foi considerado o grupo "Instituições de Ensino", o bom acesso a esse grupo de locais é mais significativo para as famílias que possuem familiares em idade escolar ou em período de formação técnico acadêmica. Os especialistas consideraram as escolas urbanas mais importantes que as escolas rurais por ter educação até o ensino médio, o que não ocorre com as rurais que possuem somente até o ensino fundamental.
Por fim, o grupo "Locais de serviços e lazer" teve um grau de importância menor na análise, pois os avaliadores consideraram que para essa funcionalidade os deslocamentos não ocorrem com a mesma frequência como para os outros locais avaliados. Verificou-se que a população rural, em termos de hábitos não apresenta as mesmas expectativas em relação a estes locais que os moradores urbanos.

Observa-se que para os especialistas, as distâncias máximas consideradas adequadas para os deslocamentos das propriedades aos LI tiveram uma variação considerável entre os grupos. Os valores admitidos de Dmáx para os locais de comercialização da produção agropecuária foram os maiores dentre os grupos, enquanto que para a educação foram os menores. Isso pode estar relacionado com o fato de que para comercializar a produção, os produtores comumente utilizam modos de transportes motorizados, como caminhão ou automóvel, e apesar da frequência, possuem maior autonomia para realizarem os deslocamentos.

Já para os deslocamentos aos locais de educação, entende-se que principalmente as escolas rurais devam estar as mais próximas e acessíveis possíveis, então a distância máxima de $5 \mathrm{~km}$ considerada adequada, pode melhor representar a acessibilidade para este tipo de escola no meio rural, já que se trata de deslocamentos frequentes dos alunos e em sua maioria a pé ou dependentes de TPU. Nesse sentido, os postos de saúde rurais também obtiveram valores menores de Dmáx $(10 \mathrm{~km})$, pois a proposta é exatamente levar às comunidades rurais atendimento médico mais próximo.

\subsection{Definição e cálculo das distâncias-custo normalizadas}

A acessibilidade incorpora valores de distâncias realizadas nos deslocamentos entre as propriedades rurais e os LI, além de fatores que contribuem como uma impedância no deslocamento. De modo a melhor representar a população estu- 


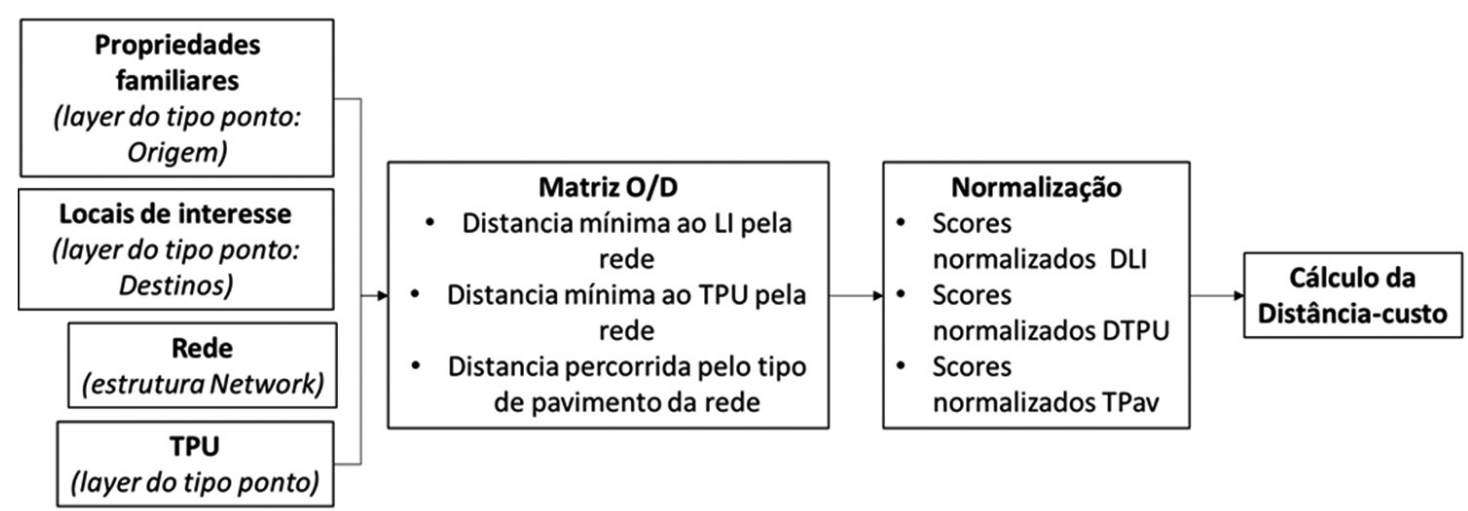

Figura 2: Determinação das distâncias-custo

dada, adotaram-se algumas distâncias que foram consideradas mais representativas em termos de acesso, verificadas durante a pesquisa em campo e apontadas pelos entrevistados. São elas:

- Distâncias das propriedades rurais aos locais de interesse (DLI): Propriedades mais distantes de seus locais de interesse possuem uma desvantagem de localização, pois requerem maiores custos com transportes e assim, possibilidades mais reduzidas de oportunidades.

- Distâncias ao transporte público (DTP): propriedades mais distantes das linhas de ônibus possuem maiores dificuldades de acesso ao TPU e consequentemente aos LI.

- Tipo de via (TPav): Estradas pavimentadas possuem vantagens de deslocamento em termos de velocidades e facilidades sobre as vias não pavimentadas, pois as estradas de terra são mais passíveis de causar transtornos, seja devido à falta de manutenção ou pela intensificação dos problemas na época de chuva, tais como atoleiros no leito da estrada com falta de canais de drenagem, desmoronamentos e quedas de barreiras.

Depois de definidos os fatores, calcularam-se as distâncias-custo sendo que a maior contribuição (80\%) é dada pela distância das propriedades rurais aos locais de interesse (DLI) considerando um fator de impedância representada pelo tipo de pavimento das vias e a menor contribuição (20\%) é dada pela distância das propriedades ao transporte público (DTP). O fluxograma da Figura 2 apresenta as etapas realizadas com o auxílio do SIG para a determinação das distâncias-custo.

O cálculo de cada distância foi realizado por meio de matrizes O/D (origem-destino), obtidas com o auxílio dos SIG ArcGIS e TransCAD, considerando a menor distância possível pela rede viária. As ferramentas utilizadas no SIG auxiliaram ainda na verificação e correção da conectividade da rede (nós e arcos da rede); conexão dos pontos de origem (propriedades) e dos LI à rede, conectando os pontos ao vértice ou à linha mais próxima. A figura $3 \mathrm{a}$ apresenta o município de Itajubá com os 52 LI, as 132 propriedades rurais e as linhas de ônibus, já a Figura 3 b apresenta as vias de acesso com relação a existência ou não de pavimento.

Com os valores das distâncias obtidas nas matrizes O/D de LI, foram selecionadas as distâncias médias ou mí-

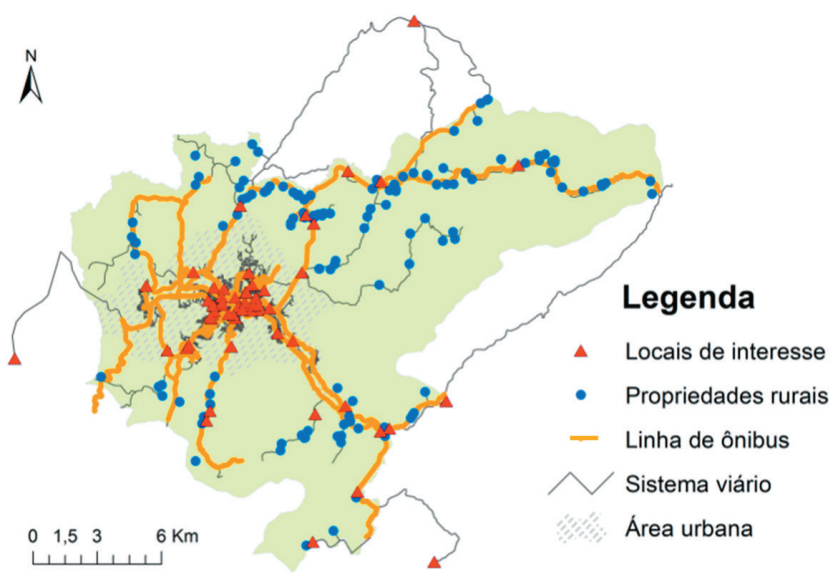

a) Linha de ônibus (TPU)

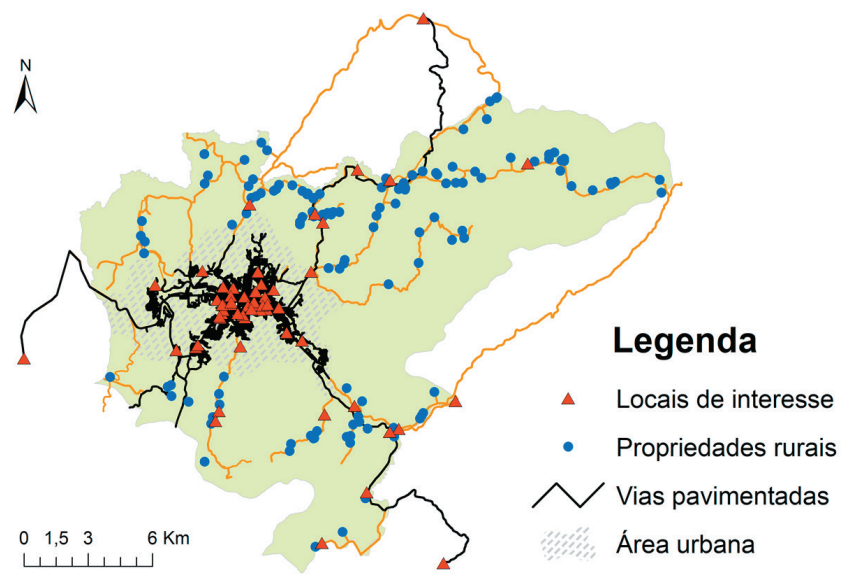

b) Vias pavimentadas e não pavimentadas

Figura 3: Localização dos LI, propriedades rurais, TPU e TPav no município.

nimas, de acordo com o motivo da viagem de cada LI (por exemplo, para escolas rurais foram selecionadas as menores distâncias da propriedade a cada escola analisada e utilizou-se o valor mínimo). Estas distâncias, bem como as distâncias ao transporte público foram normalizadas para a mesma escala de valores ( 0 a 1), utilizando funções fuzzy. Para os estabelecimentos de comercialização da produção agropecuária foram utilizadas a função linear decrescente, para os demais grupos a função utilizada foi a sigmoidal decrescente. Ainda, cada função contou com pontos de controle (Dmáx) que foram definidos pelos especialistas, conforme apresentado na Tabela 3, item 4.1. 


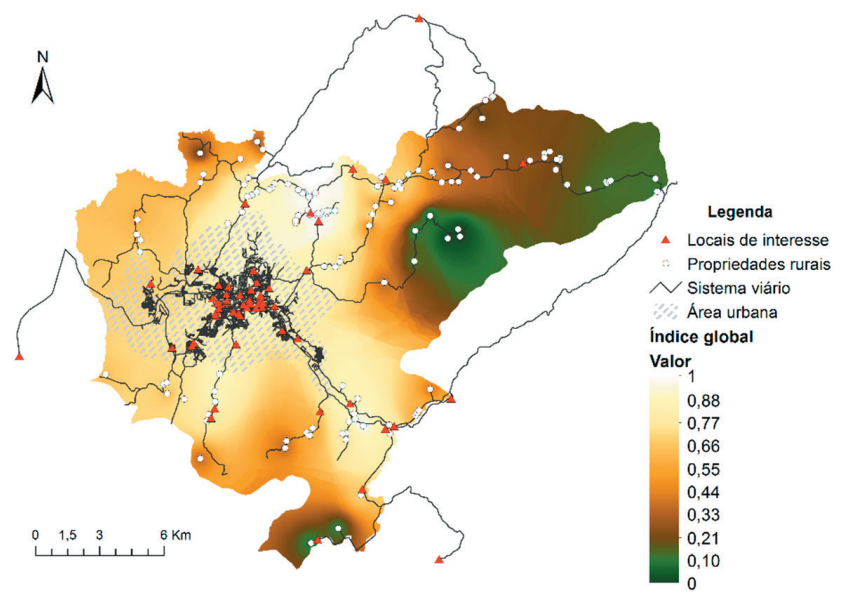

Figura 4: Acessibilidade Global aos Locais de Interesse

A Dmáx ao TPU considerada adequada foi de $2,5 \mathrm{~km}$, significando que deslocamentos a pé superiores a estas distâncias são inviáveis de serem praticados. Na literatura constatase que a distância de caminhada a pé considerada adequada no dia a dia, é inferior a 2 ou $3 \mathrm{~km}$ entre a residência e o ponto da estrada onde passa o veículo (Geipot, 1995; Vasconcellos, 1997; Pegoretti, 2005). Assim, a distância ao TPU contribuiu no cálculo do índice, reduzindo seus valores para as propriedades quanto mais excedessem essa distância máxima.

Em seguida, com o auxílio do ArcGIS Network foi possível verificar as vias pavimentadas e não pavimentadas. Foi adotado um score conforme a existência ou não de pavimento das vias percorridas, de cada propriedade para cada LI, variando desde vias $100 \%$ pavimentadas, que se referem às vias com maiores facilidades de deslocamento, passando por vias com determinadas porcentagens com pavimento e sem pavimento, até vias $100 \%$ não pavimentadas (estradas de terra), indicando maior impedância ao deslocamento.

\subsection{Cálculo do índice de acessibilidade em ambiente SIG}

Ao definir o grau de importância dos LI e o cálculo das distância-custo para cada local, parte-se ao desenvolvimento do Índice de Acessibilidade. O índice de acessibili- dade é dado pelas Equações 1, 2 e 3 apresentadas no item 3 , resultando numa avaliação multicriterial da acessibilidade das propriedades rurais aos LI, pela agregação (soma ponderada) dos valores normalizados das distância-custo e os pesos dos LI. Determinou-se a acessibilidade para cada grupo de LI e depois a acessibilidade global, agregando os 4 grupos. Os resultados preliminares já determinados por Sakamoto e Lima (2015) e os apresentados na Figura 4 expressam o grau de acessibilidade que varia no intervalo $[0,0$ a 1,0$]$, indicando uma variação contínua do índice de acessibilidade entre 0,0 (sem acessibilidade) e 1,0 (máxima acessibilidade) no território. O uso do SIG possibilitou a geração de mapas de acessibilidade, obtidos em função dos índices de cada propriedade. Foi utilizado o método de interpolação de pontos, por meio da ferramenta do Spatial Analyst Interpolation, que representa uma superfície que cobre a área de estudo não amostrada a partir dos locais amostrados (propriedades), conforme apresentado na Figura 4.

\subsection{Combinação da Acessibilidade com o relevo do território}

Com a obtenção dos mapas de acessibilidade das propriedades para cada grupo de LI e a acessibilidade global, conforme o item 5.3 e apresentados por Sakamoto e Lima (2015), realizou-se uma nova modelagem e análise dos índices a todo território considerando a declividade como fator de fricção da superfície do terreno, pois de uma forma geral, na área rural estudada as estradas de terra acompanham o relevo do terreno. O mapa de fricção representa o efeito impeditivo do relevo à facilidade de deslocamento, ou seja, quanto maior a declividade, maior a dificuldade de deslocamento.

A Figura 5 apresenta a síntese das etapas realizadas para obter os mapas finais de acessibilidade, utilizando as ferramentas da plataforma SIG.

O mapa de declividade foi obtido a partir do modelo digital de elevação (MDE) do IBGE (2010), no formato raster. Por meio da ferramenta Slope (Spatial Analyst), foi adotado o percentual de declividade, que na região de estudo obteve uma variação de $0,00 \%$ a $117,39 \%$ de declividade. Foram consideradas seis classes de declives confor-

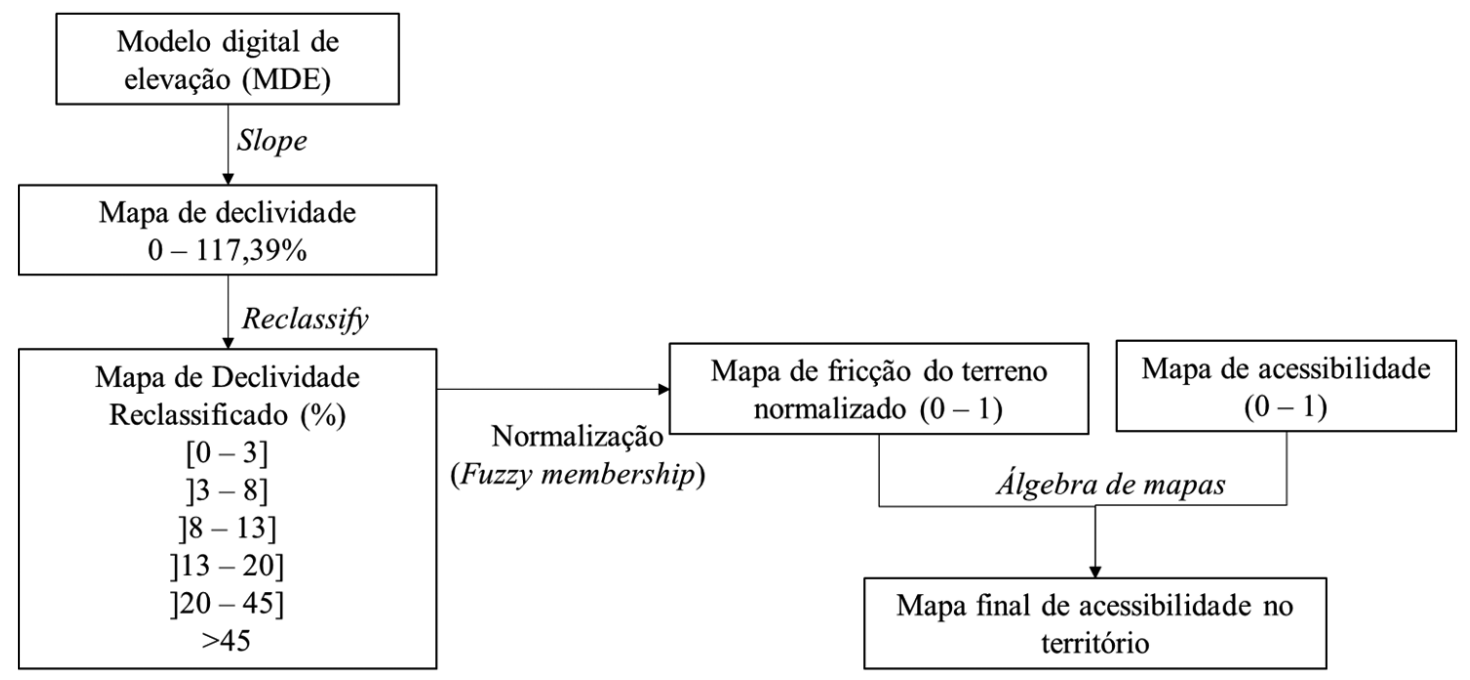

Figura 5: Etapas realizadas na obtenção do índice final de acessibilidade 


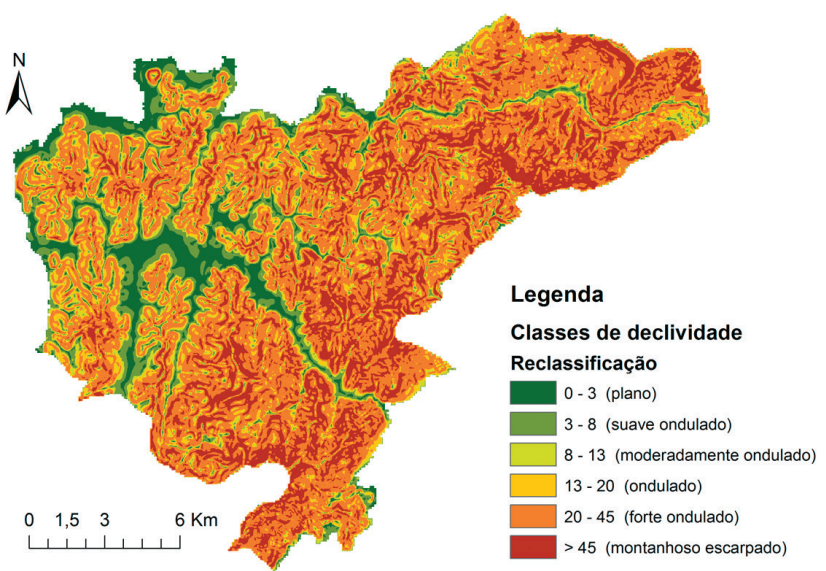

Figura 6: Mapa de declividade reclassificado.

me a classificação da EMBRAPA (Ramalho-Filho \& Beek, 1995). Segundo a Companhia de Pesquisas em Recursos Minerais (CPRM), os mapas temáticos de classes de declividade se destacam como uma ferramenta na implantação de bases de dados para tomada de decisões, sendo também um importante instrumento de análise ambiental para o estudo do espaço geográfico de modo integrado, auxiliando na detecção de áreas críticas para deslizamento de encostas e permitindo diversas análises de terreno, como no planejamento de lavouras agrícolas e na identificação de áreas suscetíveis à ocorrência de erosões e inundações (CPRM, 2010). Assim, o mapa da Figura 6 apresenta as seis classses de declives consideradas na análise, obtidas pela ferramenta Reclassify.

Observa-se que o relevo é bastante diversificado, em grande parte da área ele é fortemente ondulado e montanhoso, nessas áreas a declividade varia de $20 \%$ a $45 \%$ e acima de 45\%, respectivamente. Segundo Pereira (2004) a declividade é um dos fatores relacionados aos processos de suscetibilidade à erosão e de forma geral, além de outras diversas características, as terras que possuem maior suscetibilidade ocorrem em relevo ondulado a montanhoso $(>13 \%)$. As áreas mais planas são as que circundam os principais rios, com destaque a bacia do Rio Sapucaí, Lourenço Velho e Ribeirão Anhumas, áreas também de maior ocupação antrópica no território.

O mapa de declividade foi novamente reclassificado numa escala de 0 a 1 , sendo 1 a melhor condição de declividade para o deslocamento, representando as áreas mais planas e zero para áreas não aptas para o deslocamento, áreas com maiores declividades. Foi utilizada a ferramenta "Fuzzy membership" com uma função linear, de forma que a partir de $45 \%$ de declividade fossem adotados valores 0 , representando áreas com pior condição de declividade para o relevo montanhoso escarpado. Por fim, a partir do mapa de fricção normalizado e dos mapas de acessibilidade obtêm-se os mapas finais, com um novo índice de acessibilidade para a totalidade do território em análise. Para tal, foi utilizada a calculadora raster da álgebra de mapas, com o cálculo da média entre os dois mapas, de fricção e de acessibilidade, para cada grupo e o global, como apresentado nos mapas da Figura 7, que representam a acessibilidade no território pelos diferentes grupos de LI e o global para todos os LI.

A análise de um novo índice de acessibilidade do território, considerando a fricção permitiu uma leitura mais compatível com a realidade de estudo, já que o relevo é um dos

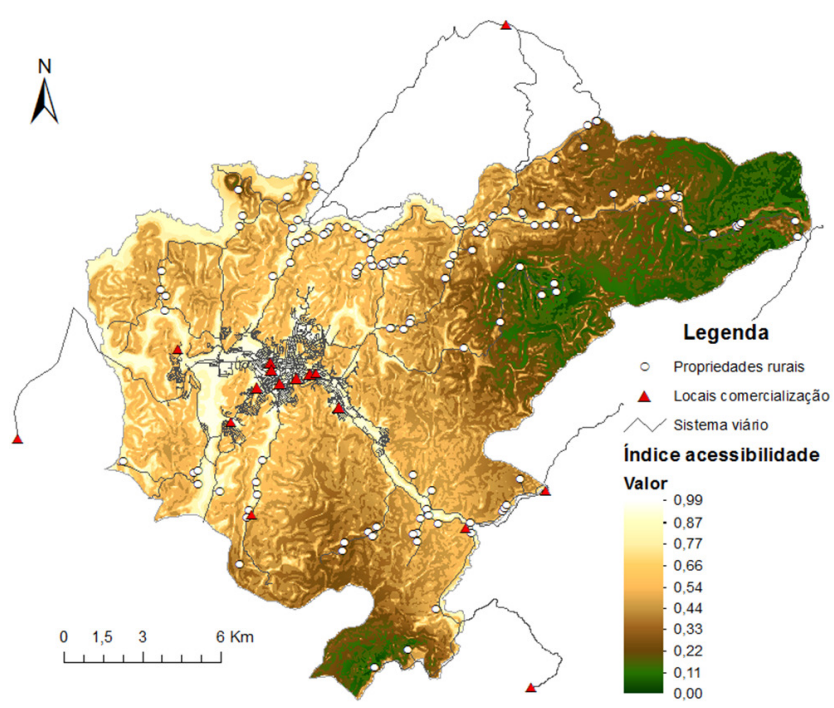

a) Comercialização da produção

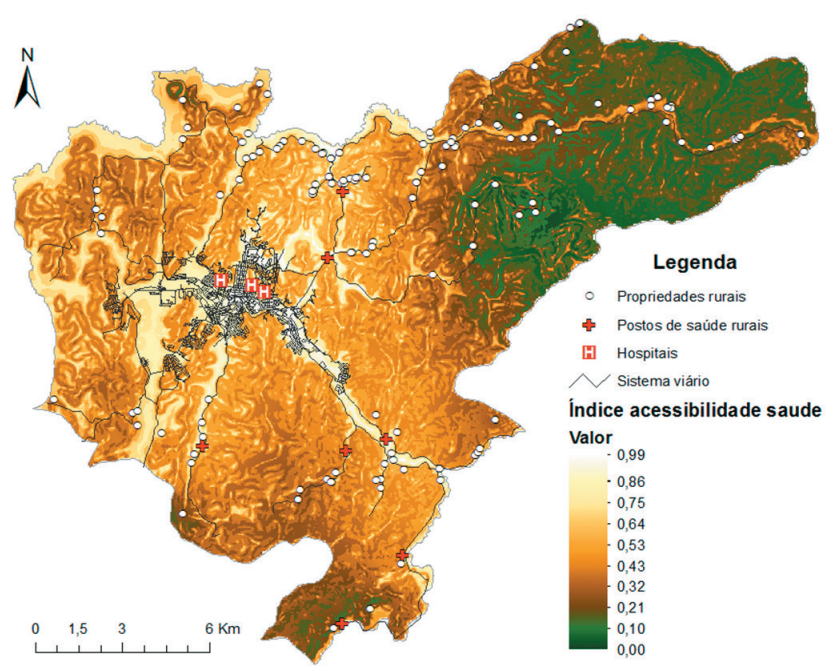

b) Equipamentos de saúde

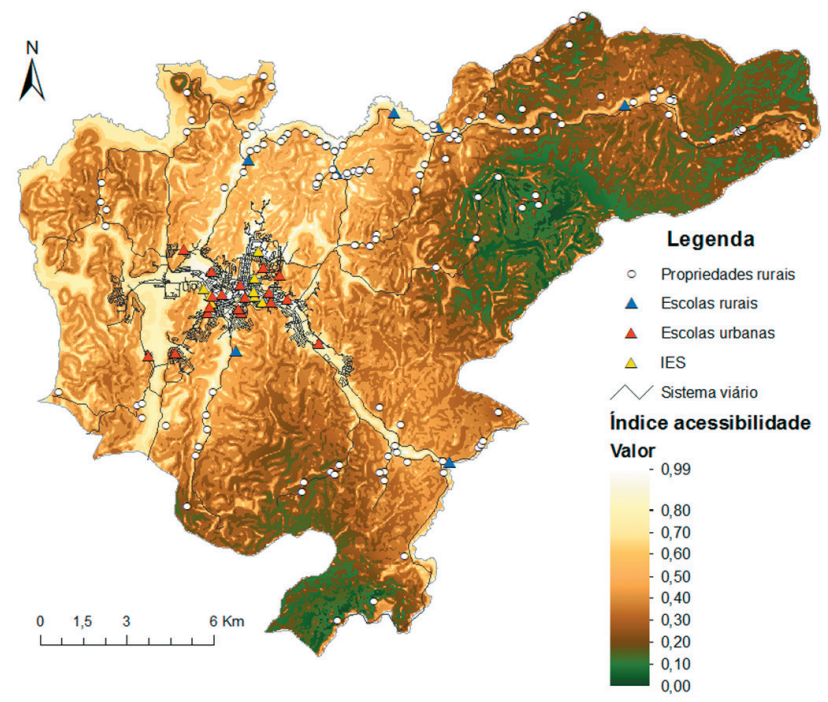

c) Instituições de ensino 


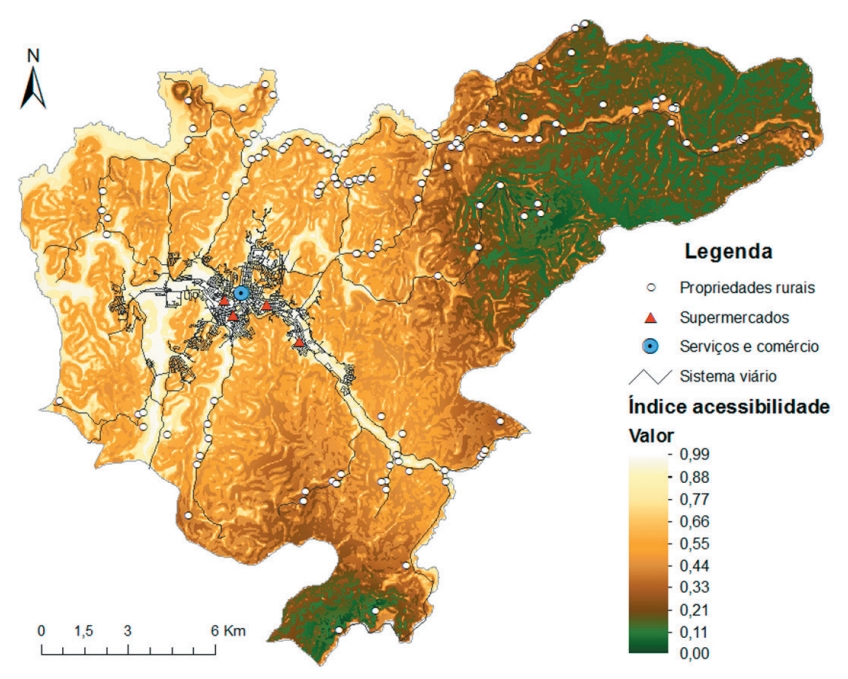

d) Locais de Serviços e lazer

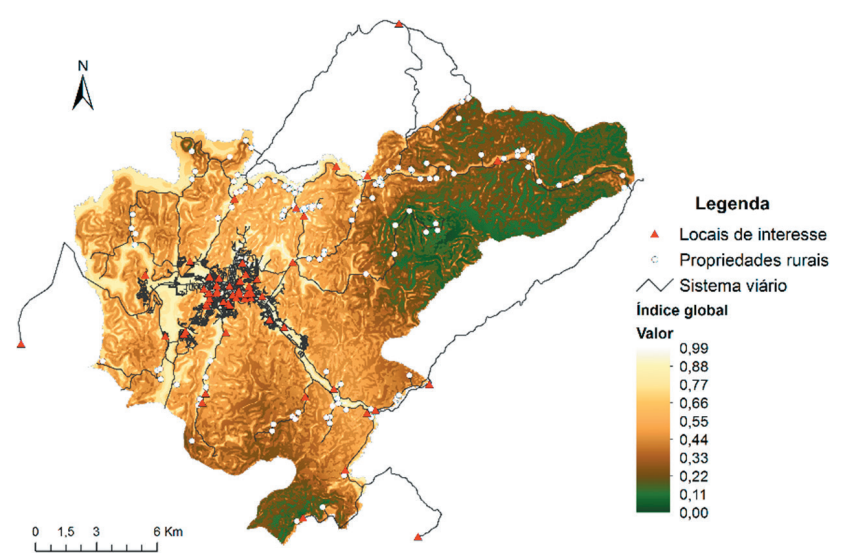

e) Mapa de acessibilidade global do território

Figura 7: Mapas de acessibilidade aos grupos de LI e global

fatores que interferem nas condições de acesso. Ao incorporar essa dificuldade dada pela declividade, verificou-se de forma geral, para todos os grupos e no mapa global que os novos índices adquiriram valores menores por causa da declividade.

Pela análise dos mapas da Figura 7, constata-se que a acessibilidade aos LI para todos os grupos, são mais elevados para as propriedades que se localizam na região central e oeste do município, tanto por se tratar de áreas mais planas, principalmente ao que refere às áreas próximas aos principais rios existentes, com índices próximos à 0,99 , quanto pela maior proximidade aos LI considerados, e por esta localização comumente ser privilegiada com acesso fácil ao TPU e com vias pavimentadas, melhorando as condições de acesso e o tempo de deslocamento. Entretanto, verifica-se que os LIs que obtiveram maior peso dentro de cada grupo, tais como laticínios, escolas rurais, postos de saúde rurais, mesmo quando se encontram em áreas rurais mais afastadas da região central, também contribuíram para que naquela região a acessibilidade melhorasse.

As propriedades com os piores índices de acessibilidades são as da região nordeste e sul do município, com índices chegando próximos à zero, indicando a falta de acessibilidade para estas áreas, que além de apresentar o relevo em sua maioria montanhoso e escarpado, não possui proximidades aos LI e ao transporte público, sendo que e o percurso ocorre em sua maioria por estradas de terra, que dependem da realização de manutenções periódicas para que apresentem boas condições de acesso, sendo que essas condições tendem a piorar na época de chuvas, podendo ter relação também com os aspectos do relevo, como os problemas oriundos da alta susceptibilidade à erosão, podendo dificultar e até impossibilitar o deslocamento aos LI. Nesse sentido, essas propriedades apresentam uma desvantagem em relação às outras melhores localizadas, tanto em relação aos maiores custos com transportes, quanto pelas menores oportunidades.

Ao realizar uma comparação da acessibilidade entre os grupos, é possível notar que para os grupos "Comercialização da produção" e "Locais de Serviços e lazer" a acessibilidade relativamente melhora, e a razão principal se dá em função de os LI se situarem a distâncias menores do que as consideradas na função para a normalização. Ao passo que para os grupos "Equipamentos de saúde" e "Instituições de ensino", a acessibilidade no território foi menor porque as distâncias encontradas na região são superiores aos valores adotados como pontos de controle (por exemplo, Dmáx de $10 \mathrm{~km}$ para os postos rurais e $5 \mathrm{~km}$ para escolas rurais).

\section{CONCLUSÃO}

Este trabalho apresentou a avaliação da acessibilidade em ambiente SIG e, o uso do SIG possibilitou a geração de mapas e um melhor entendimento em termos de distribuição geográfica e da variação do índice de acessibilidade no território. A coleta de dados por meio de entrevistas, questionários e uso de um GPS portátil em campo se mostraram essenciais na obtenção de informações referentes aos hábitos e padrões de viagens das famílias, permitindo estabelecer uma lista dos principais locais de interesse dos agricultores familiares em termos de comercialização da produção, educação, saúde, serviços e lazer.

A associação da metodologia multicritério ao SIG permitiu incorporar na análise, fatores como as distâncias aos principais locais de interesse, o tipo de pavimento existente nas vias e o acesso ao TPU, levando em consideração o julgamento de especialistas quanto ao grau de importância de cada local. Dessa forma, foi possível verificar a acessibilidade das propriedades aos diferentes tipos de locais de interesse, de forma que possibilitou a comparação dos mapas de acessibilidade pelas diferentes funcionalidades.

Os grupos locais de comercialização da produção agropecuária e equipamentos de saúde foram os que obtiveram os maiores pesos na análise, considerando que os deslocamentos para comercialização têm fundamental importância na aquisição de renda e sustento familiar e, o serviço público de saúde é tido como essencial para a melhora das condições de vida da população rural.

A região está inserida na bacia hidrográfica do Sapucaí na Serra da Mantiqueira, possuindo em maior parte do território relevo fortemente ondulado e montanhoso. Por este motivo, a análise da acessibilidade do território considerando a fricção (pela declividade do terreno), permitiu uma leitura mais compatível com a realidade de estudo, já que o relevo é um dos fatores que interfere nas condições de acesso. A avaliação da acessibilidade mostrou que há diferença de acessibilidade entre as propriedades estudadas. 
Os locais mais acessíveis foram os mais próximos à região central e oeste do município, que são áreas mais planas e onde se localizam a maioria dos locais de interesse e também onde há maior presença de vias pavimentadas e transporte coletivo. Os piores índices foram observados nas regiões nordeste e sul do município, o que indica que essas áreas necessitam de maior atenção em termos de planejamentos e melhorias para a acessibilidade, seja em termos de infraestruturas ou transportes. Estes locais são mais isolados e possuem pouca oferta de equipamentos e infraestruturas sociais básicas (saúde, educação, e transporte público), e aonde também os deslocamentos ocorrem em sua maioria por meio de estradas sem pavimento, que por se encontrarem em áreas de maior declividade, sofrem com o efeito impeditivo do relevo à facilidade de deslocamento e com as condições das estradas.

Finalmente, conclui-se que a acessibilidade no meio rural não pode ser vista somente como forma de acesso aos mercados para comercialização e obtenção de renda, mas também como forma de permitir ao agricultor uma maior autonomia em relação a esta dependência dos mercados e fatores externos, no sentido de também proporcionar melhores condições de vida no meio rural, em termos de saúde, educação, segurança alimentar etc., permitindo assim, um desenvolvimento mais sustentável e equitativo no território.

\section{REFERÊNCIAS}

Abramovay, R. (1998) Agricultura familiar e desenvolvimento territorial. Reforma agrária, v. 28, n. 1, p. 2.

Abramovay, R. (2003) O futuro das regiões rurais. Porto Alegre: Editora da UFRGS.

Aguiar, F. O. (2010). Acessibilidade relativa dos espaços urbanos para pedestres com restrições de mobilidade. Tese (Doutorado) - Escola de Engenharia de São Carlos da Universidade de São Paulo. Disponível em: < http://disciplinas. stoa.usp.br/pluginfile.php/151701/mod_resource/content/3/ tese-acessibilidade $\% 20 \mathrm{em} \% 20$ espa $\% \mathrm{C} 3 \% \mathrm{~A} 7 \mathrm{os} \% 20$ urbanos. pdf $>$ Acesso em: 8 mar. 2016.

Allen, W. B.; D. Liu E Singer, S. (1993). Accessibility measures of U.S. metropolitan areas. Transportation Research, Part B, Methodological, v. 27, n. 6, p. 439-50. DOI: 10.1016/0191-2615(93)90016-4

Brasil (2006). Lei n. 11.326, de 24 de julho de 2006. Estabelece as diretrizes para a formulação da Política Nacional da Agricultura Familiar e Empreendimentos Familiares Rurais. Presidência da República. Brasília, DF. Disponível em: $<$ http// planalto.gov.br/ccivil_03/_ato2004-2006/2006/lei/111326.htm>. Acesso em: 6 jul. 2014.

CPRM - Companhia de Pesquisas em Recursos Minerais Serviço Geológico do Brasil (2010). Mapa de declividade em percentual do relevo brasileiro. Disponível em: <http://www. cprm.gov.br/publique/Gestao-Territorial/Geodiversidade/ Mapa-de-Declividade-em-Percentual-do-RelevoBrasileiro-3497.html >. Acesso em: 10 maio 2015.
IBGE - Instituto Brasileiro de Geografia e Estatística (2009). Censo agropecuário 2006. agricultura familiar: primeiros resultados: Brasil, grandes regiões e unidades da federação. Brasília/Rio de Janeiro: MDA/MPOG.

IBGE - Instituto Brasileiro de Geografia e Estatística (2010). Censo Demográfico 2010: características gerais da população. Rio de Janeiro, RJ, Brasil.

IFAD - International Fund for Agricultural Development (2010). Rural poverty report 2011: new realities. New Challenges: New Opportunities for Tomorrows Generation, International Fund for Agricultural Development, p. 16.

Ingram, D. R. (1971). The concept of accessibility: a search for an operational form. Regional Studies, v. 5, n. 2, p. 101-7. DOI: 10.1080/09595237100185131.

Geipot (1995). Avaliação preliminar do transporte rural: destaque para o segmento escolar. Brasília, DF: Empresa Brasileira de Planejamento de Transportes.

Kageyama, A. (2004). Desenvolvimento rural: conceito e medida. Cadernos de Ciência \& Tecnologia. Brasília, v. 21, n. 3, p. 379-408.

Lebo, J., Schelling, D. (2001). Design and appraisal of rural transport infrastructure: ensuring basic access for rural communities. World Bank Technical Report, n. 496, p. 4.

Lima, J. P. (2007). Modelo de decisão para a priorização de vias candidatas às atividades de manutenção e reabilitação de pavimentos. Tese (Doutorado) - Escola de Engenharia de São Carlos, Universidade de São Paulo. São Carlos, SP.

Lima, J. P.; Ramos, R. A. R.; Fernandes Júnior, J. L. (2009). Uma abordagem multicritério para a priorização de vias pavimentadas. Transportes, v. 17, n. 1, p. 27-38. DOI: 10.14295/ transportes.v17i1.73.

Machado, M. H.; Lima, J. P.(2015). Avaliação multicritério da acessibilidade de pessoas com mobilidade reduzida: um estudo na região central de Itajubá (MG). Urbe. Revista Brasileira de Gestão Urbana, p. 368-382. DOI: 10.1590/2175-3369.007.003.AO08.

Malczewski, J. (1999). GIS and multicriteria decision analysis. New York: John Wiley \& Sons, Inc.

Maluf, R. S. (2002). O enfoque da multifuncionalidade da agricultura: aspectos analíticos e questões de pesquisa. In: Lima, D.M.; Wilkinson, J. (Org.) Inovação nas tradições da agricultura familiar. Brasília: CNPq/Paralelo 15, p. 301-328.

MDA/SDT- Ministério do Desenvolvimento Agrário Secretaria de Desenvolvimento Territorial (2003). Referências conceituais do desenvolvimento territorial rural sustentável. Brasília, DF: CONDRAF/NEAD.

Mendes, J. F. G. (2001). Multicriteria accessibility evaluation using gis as applied to industrial location in Portugal. Earth Observation Magazine, v. 10, n. 2, p. 31-35. 
Ministério das Cidades (2006). Programa Brasil acessível: programa brasileiro de acessibilidade urbana: cadernos 1 a 6 . Brasília. Disponível em <http://www.cidades.gov.br/institucional/ biblioteca-acesso/138-secretaria-nacional-de-transporte-e-damobilidade/3409-biblioteca>. Acesso em: 5 set. 2015.

Miranda, L. I. B. (2011). Planejamento em áreas de transição rural-urbana: velhas novidades em novos territórios. Revista Brasileira de Estudos Urbanos e Regionais, v. 11, n. 1, p. 25-40.

Morris, J. M.; Dumble, P. L.; Wigan, M. R. (1979). Accessibility indicators for transport planning. Transportation Research, Part A, v. 13, n. 2, p. 91-109. DOI: 10.1016/0191-2607(79)90012-8.

Oliveira, G. M.; da Silva A. N. R. (2015). Desafios e perspectivas para avaliação e melhoria da mobilidade urbana sustentável: um estudo comparativo de municípios brasileiros. Transportes, v. 23, n. 1, p. 59-68. DOI: 10.4237/transportes.v23i1.768.

Pegoretti, M. S. (2005). Definição de um indicador para avaliar a acessibilidade dos alunos da zona rural às escolas da zona urbana. Dissertação (Mestrado) - Escola de Engenharia de São Carlos, Universidade Federal de São Carlos. São Carlos, SP.

Pegoretti, M. S.; Sanches, S. P. (2006). Transporte escolar: indicador de acessibilidade dos alunos da zona rural às escolas da zona urbana. Revista dos Transportes Públicos, v. 110, p. 83-98.

Pereira, L. C., Neto, F. L. (2004). Avaliação da aptidão agrícola das terras: proposta metodológica. Jaguariúna: Embrapa Meio Ambiente, 43. $36 \mathrm{p}$.

Raia Junior, A. A. (2000). Acessibilidade e mobilidade na estimativa de um indice de potencial de viagens utilizando redes neurais artificiais e sistemas de informações geográficas. Tese (Doutorado) - Escola de Engenharia de São Carlos, da Universidade de São Paulo. São Carlos, SP.

Ramalho-Filho, A., Beek, K. J. (1995). Sistema de avaliação da aptidão agrícola das terras. 3. ed. Rio de Janeiro: EMBRAPACNPS.

Rodrigues, D. S. (2001). Avaliação Multicritério de Acessibilidade em Ambiente SIG. Dissertação (Mestrado) Escola de Engenharia, Universidade do Minho. Braga, Portugal.

Rodrigues, D. S., Mendes, J. F. G., Lima, J. P., Ramos, R. A. R. (2004). Uma abordagem multicritério para avaliação da acessibilidade. In: Mendes, J. F. G.; Silva, A. N. R. Souza, L. C. L.; Ramos, R. A. R. (Org.). Contribuições para o desenvolvimento sustentável em cidades portuguesas e brasileiras. 1. ed. Coimbra, Portugal: Almedina, v. 1, p. 98-117.

Saaty, T. L. (2008). Decision making with the analytic hierarchy process. International Journal of Services Sciences, v. 1, n. 1, p. 83-98. DOI: 10.1504/IJSSci.2008.01759.

Sachs, I. (2002). Redescoberta e invenção do Brasil rural. Brasília: Conselho Nacional de Desenvolvimento Rural Sustentável-CNDRS.
Sakamoto, E. (2015). Avaliação multicritério da acessibilidade de agricultores familiares no município de Itajubá-MG. Dissertação (Mestrado). Universidade Federal de Itajubá. Itajubá-MG. Disponível em: $<$ http:// repositorio.unifei.edu.br:8080/xmlui/handle/123456789/167>

Santos, M. (2008). O espaço dividido: os dois circuitos da economia urbana dos países subdesenvolvidos. 2. ed. São Paulo: Ed. da USP.

Tobias, M. S. G., Ramos, R. A. R., Ravena, N., Rodrigues, D. S. (2012). Aspectos da mobilidade urbana em cidades do Baixo Amazonas: indicadores para elaboração de políticas públicas. Revista dos Transportes Públicos, v. 129, p. 71-84.

Vasconcellos, E. A. (1997). Transporte rural: o resgate de um tema esquecido. Revista dos Transportes Públicos, v. 75, p. 31-48.

Vasconcellos, E. A. (2000). Transporte urbano nos países em desenvolvimento: reflexões e propostas. 3. ed. São Paulo: Annablume.

Veiga, J. E. (2007). Mudanças nas relações entre espaços rurais e urbanos. Revista Brasileira de Gestão e Desenvolvimento Regional, v. 3, n. 1. 